\title{
hTERT promoter mutations in chondrosarcomas associate with progression and disease-related mortality
}

\author{
Yingbo Lin ${ }^{1} \cdot$ Nelly Seger $^{1} \cdot$ Yi Chen ${ }^{1} \cdot$ Asle C. Hesla ${ }^{2,3} \cdot$ Johan Wejde ${ }^{1,4} \cdot$ Mehran Ghaderi $^{1,4} \cdot$ Panagiotis Tsagkozis ${ }^{2,3}$. \\ Olle Larsson ${ }^{1,4} \cdot$ Felix Haglund $\mathbb{B}^{1,4}$
}

Received: 5 February 2018 / Revised: 28 May 2018 / Accepted: 29 May 2018 / Published online: 31 July 2018

(c) United States \& Canadian Academy of Pathology 2018

\begin{abstract}
Chondrosarcomas are malignant skeletal tumors with chondroid differentiation. Prognosis is largely dependent on histological grading, which suffer from significant interobserver variability. Telomerase activity and abundant telomerase reverse transcriptase ( $h T E R T$ ) expression has previously been associated with chondrosarcoma grade and metastasis. We therefore analyzed the $h T E R T$ promoter in clinicopathologically well-characterized chondrosarcomas (grade 1-3) from 87 patients. Using Sanger sequencing we identified an activating $-124 \mathrm{C}>\mathrm{T}$ mutation in 23 cases (26\%). Promoter mutations were significantly associated with increased histological grade ( $8 \%$ of grade $1,32 \%$ of grade 2 and $46 \%$ of grade $3, P=$ 0.002), suggesting a role in tumor progression. In four chondrosarcomas where the histopathological grade was heterogenous, the $h T E R T$ mutation was only identified in the higher-grade areas. Additionally, $h T E R T$ promoter mutations were significantly associated with worse metastasis-free survival $(P=0.018)$, chondrosarcoma-specific survival $(P=0.022)$ and older patient age $(P=0.003)$. These data suggest that $h T E R T$ promoter mutations are common in high grade conventional chondrosarcomas. Granted that additional studies can confirm these findings; $h T E R T$ promoter analysis could potentially serve as an adjuvant prognostic marker in routine chondrosarcoma grading. This study reinforces the rationale of telomerase targeted therapy in a subset of chondrosarcomas.
\end{abstract}

\section{Introduction}

Chondrosarcomas are malignant mesenchymal tumors with cartilaginous differentiation. The majority are conventional chondrosarcomas which arise de novo at the axial or

Precís: We characterized the hTERT promoter in chondrosarcomas and found recurrent activating mutations which was significantly linked to progression and mortality.

Felix Haglund

Felix.Haglund@ki.se

1 Department of Oncology-Pathology, Cancer Centre Karolinska, Karolinska Institutet, Stockholm, Sweden

2 Department of Molecular Medicine and Surgery, Karolinska Institutet, Stockholm, Sweden

3 Department of Orthopedic surgery, Karolinska University Hospital Solna, Stockholm, Sweden

4 Department of Clinical Pathology and Cytology, Karolinska University Hospital Solna, Stockholm, Sweden appendicular skeleton. Occasionally secondary chondrosarcomas may arise from preexisting chondroid lesions such as enchondromas or osteochondromas. To assess the clinical behavior of chondrosarcomas, tumors are classified in conjunction with radiological, clinical, and pathological evaluations.

Histologically, chondrosarcomas are graded according to the architectural and cytological atypia [1]. Grade 1 (lowgrade) tumors are dominated by lobulated chondroid hyaline matrix and scattered chondrocytes with mild nuclear enlargement and multinucleation in lacunae, which may be indistinguishable from benign enchondromas. Additionally, low grade chondrosarcoma can have confluent growth pattern, host bone entrapment, skip lesion growth into Haversian canal, and soft tissue involvement (more than two-thirds scalloping of the cortex) [2]. Grade 2 (intermediate) chondrosarcomas are more cellular with loss of lobulated architecture, myxoid change, and increased cellularity. Grade 3 (high-grade) chondrosarcomas are highly cellular with severe nuclear atypia and peripheral spindle cell morphology. These also may exhibit mitotic activity and extensive necrosis. Chondrosarcoma, usually of low 
grade, may undergo dedifferentiation to a pleomorphic spindle cell tumor that resembles undifferentiated pleomorphic sarcoma, outside of the hyaline cartilage lobules (sometimes referred to as grade 4). While grading is currently the best predictor of patient outcome, the histological assessment is subjective with significant interobserver variability $[3,4]$. There is still a lack of any effective systemic treatment for metastatic disease [5]. Naturally, there is a strong need for objective prognostic markers and potential therapeutic targets in chondrosarcomas.

Patients with Olliers and Maffucci syndromes harbor mosaic mutations in the $I D H I$ and $I D H 2$ genes, which predispose for multiple enchondromas [6-8]. A subset of these lesions progress to secondary central chondrosarcomas. Recurrent mutations or deletions in the IDH1,IDH2, COL2A1, ACVR2A, and YEATS genes have been identified in both chondrosarcomas and enchondromas [9-11]. Conversely, mutations in the TP53 gene or a complex tumor karyotype has been linked to aggressive clinical behavior $[12,13]$.

Mutations in the gene promoter of human telomerase reverse transcriptase ( $h T E R T$ ) has been described in numerous cancer subtypes and are frequently associated with a more aggressive phenotype [14]. These mutations generate transcription factor binding motifs which may lead to $h T E R T$ gene overexpression, which is believed to induce telomerase activity [15-17].

Martins et al. reported $h T E R T$ expression in 7/16 chondrosarcomas and demonstrated a relationship between $h T E R T$ expression and cell proliferation, transition to more aggressive morphology and invasive behavior in chondrosarcoma cell lines [18]. Additionally, they observed that diffuse TERT immunoreactivity was significantly associated with tumor grade and metastasis [19]. Other groups have reported that hTERT positive chondrosarcoma cell lines were sensitive to telomerase inhibition and Paclitaxel treatment [20], and that the majority of clinical chondrosarcomas exhibit telomerase activity and show $h T E R T$ gene expression [21]. Two studies have investigated hTERT promoter mutations in chondrosarcomas, one reporting ten wild-type tumors, and the second reporting one mutated and one wild-type case [22, 23].

Given the limited previous reports of telomerase expression in chondrosarcomas, we hypothesized that $h T E R T$ promoter mutations could explain some chondrosarcomas with high $h T E R T$ expression. We therefore investigated the hTERT promoter in a larger group of conventional chondrosarcomas.

\section{Material and methods}

\section{Patients and tissue samples}

In this study we investigated 87 patients with conventional chondrosarcomas operated at the Karolinska University
Hospital between 2010 and 2017. Patient and tumor data were retrieved from digital charts and pathology records, including patient age and sex, tumor location, size, grade, and surgical margins. All data was available for all patients. The tumors were graded according to WHO 2013 criteria [1]. All chondrosarcomas were graded after demonstration at a multidisciplinary conference including sarcoma specialized pathologists and radiologists. Follow up data was available for all patients, and was calculated from the day of surgery until the date of event, including local recurrence, metastasis, and death. Three patients suffered from hereditary syndromes predisposing for chondroid tumors; one patient had Mb Maffuci, one patient had Mb Ollier and one patient had hereditary multiple exostoses. In five other patients there was a known preexisting osteochondroma.

\section{DNA extraction and $h T E R T$ promoter sequencing}

For each tumor, one single formalin fixed paraffin embedded (FFPE)-tumor block representing the worst histological grade was selected for DNA extraction and sequencing. For 11 patients more than one tumor was analyzed (range 2-4 tumors). Using sterile sectioning, a total of six $10 \mu \mathrm{m}$ slides were processed using the Maxwell 16 FFPE LEV DNA purification kit (\#AS1130, Promega, Madison, WI, USA) with a prolonged incubation for 3 days, on a Maxwell 16 High Strength LEV Magnetic Rod and Plunger Bar Adaptor (\# SP1070, Promega). For identification of genetic subclones (see below), 1-mm punch biopsies were processed with a similar approach. The DNA was quantified using a NanoDrop ND-1000. Since most chondrosarcomas with low cellularity (generally grade $1-2$ ) yielded low DNA concentrations $(<10 \mathrm{ng} / \mu \mathrm{l})$ we used $100 \mu \mathrm{l}$ of elution buffer to generate a larger total yield.

Polymerase chain reaction (PCR) amplification of the hTERT promoter was done using a touch-down thermocycling protocol as previously described [14]. Reactions were run in $50 \mu \mathrm{l}$ volumes using AmpliTaqGold 360 Mastermix (\#4398881, Thermo Fisher Scientific, Waltham, MS, USA) and $>10 \mathrm{ng}$ of DNA. PCR products were purified in spin columns (QIAquick PCR Purification Kit, \#28106, Qiagen, Hilden, Germany). All mutations were verified by reverse sequencing in a separate amplification PCR. Additionally, the mutations were verified in a second DNAextraction if there was enough material available (all but two cases). Purified PCR products were sequenced by GATC Biotech (Cologne, Germany) and manually analyzed using the 4Peaks Software (version 1.7.1, Mekentosj).

\section{Immunohistochemistry}

After DNA sequencing, all chondrosarcomas with identified hTERT promoter mutations were analyzed for TERT 
immunoreactivity with a monoclonal rabbit anti-TERT antibody (\#ab32020 clone Y182 from Abcam, Cambridge, UK). Heat induced antigen retrieval was performed for $10 \mathrm{~min}$ in citrate pH 6.0 (\#S2369, Dako Agilent, Santa Clara, CA, USA), and the primary antibody incubation (at 1:100 dilution) was done overnight in $4{ }^{\circ} \mathrm{C}$. FFPE human fibroblasts

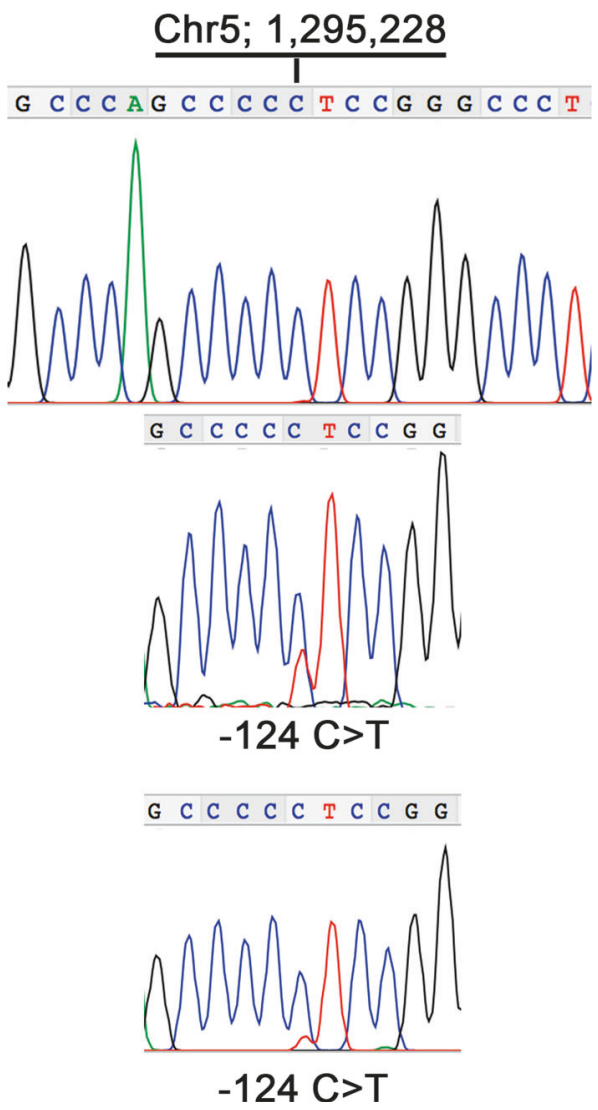

Fig. 1 Chromatograms of the wild-type (top) hTERT promoter, and two examples (middle and lower) of the $-124 \mathrm{C}>\mathrm{T}$ mutations found in chondrosarcomas. The amplitudes of the mutation curves indicate either contamination with normal DNA or a genetic subclone in the chondrosarcoma. Sequencing of punch biopsies from higher-grade tumor areas showed equivalent peak amplitude for the mutated and wild-type alleles (not shown) transfected with $h T E R T$, kindly provided by Dr. M. Ehnman at Karolinska Institutet, were used as a positive control. Untransfected fibroblasts were used as negative control.

\section{Statistics}

We used two-sided Fishers exact tests for comparisons between categorical variables and $T$-tests for comparing means between categorical variables. Overall survival, tumor-specific survival, metastasis-free survival, and risk of local recurrence were calculated from the time of surgery to the time of event. We used the Kaplan-Meier method and log-rank tests to evaluate survival differences between groups. A $P$-value below 0.05 was considered as statistically significant. All statistics were calculated using IBM SPSS (version 21, IBM Corp., NY, USA).

\section{Results}

\section{Cohort characteristics}

A total of 87 patients (50 males and 37 females) with grade 1 $(41 \%)$, grade $2(29 \%)$, or grade $3(30 \%)$ conventional chondrosarcomas were included in the study. The average tumor size was $8.2 \mathrm{~cm}$ (range 1-36 cm). Surgical margins for grade 1 were intralesional (No 20), marginal (No 10), or wide (No 6); for grade 2 they were intralesional (No 8), marginal (No 14), or wide (No 3); and for grade 3 they were intralesional (No 5), marginal (No 14), or wide (No 7). The average follow-up time was 4.4 years (range $0.12-31$ years). Six of the studied lesions were local recurrences. In total 22 patients with chondrosarcomas developed local recurrence, 12 patients developed distant metastasis and 12 patients died during follow-up (nine died of the disease). Metastatic lesions were available for analysis from four patients. The primary chondrosarcomas were located in the upper (No 18) or lower (No 27) extremities, pelvic region (No 17), thorax (No 19), or the head and neck region (No 5).
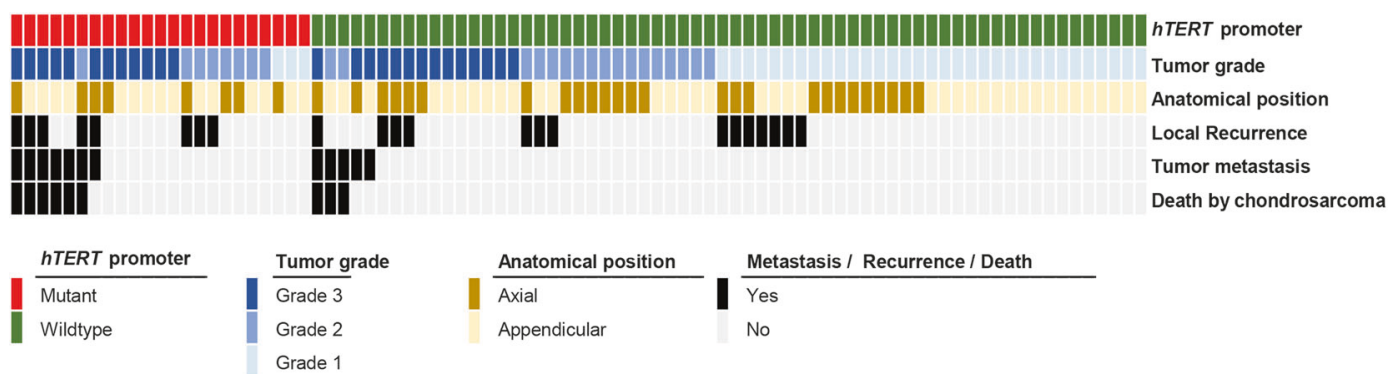

Fig. 2 Schematic overview of clinical and tumor data of the 87 individual patients 


\section{hTERT promoter mutations are associated with chondrosarcoma grade}

The previously characterized C $>\mathrm{T}-124$ hTERT promoter mutation (located at Chr5;1,295,228) was identified in 23 chondrosarcomas. Chromatograms from block sections showed low or very low mutation peaks (Fig. 1) indicating either contamination with normal DNA or subclonal events. In three patients with $h T E R T$ promoter mutated chondrosarcomas, the local recurrences showed a wild-type promoter. In a patient where the primary tumor was wild type, the metastatic lesion showed a mutation. The remaining three metastatic lesions were wild type (as their primary tumors). Finally, one wild-type tumor had a local recurrence with the mutation.

We observed a significant association between hTERT promoter mutations and increasing tumor grade in the chondrosarcomas $(P=0.002$, Fig. 2 and Table 1$)$ and older age $(P=0.003$, Table 1$)$. hTERT promoter mutation was also significantly associated with metastasis $(P=0.013)$, overall death $(P=0.013)$, and tumor associated death $(P=$ $0.009)$ at follow-up.

There was no significant association between hTERT promoter mutated chondrosarcomas and tumor size, anatomical location (axial vs appendicular), patient sex or local recurrence (Table 1).

\section{hTERT promoter mutations and chondrosarcoma patient survival}

Kaplan-Maier estimators for conventional chondrosarcomas showed that hTERT promoter mutations were significantly associated with increased risk of metastasis $(P=0.0018)$ and worse chondrosarcomaspecific survival $(P=0.022)$ as shown in Fig. 3. Tumor grading showed a strong association to risk of metastasis $(P<0.0001)$ and chondrosarcoma-specific survival $(P=$ 0.016). The limited number of patients did not allow for adequate sub-group analysis regarding tumor metastasis or patient survival. Out of 26 patients with grade 3 tumors, 12 had an hTERT mutation and 14 were wild-type. Interestingly, out of the 12 cases with mutation $43 \%$ died by disease. The corresponding disease-related mortality of the wildtype tumors was only $5 \%$, suggesting that $h T E R T$ promoter status may be a useful prognostic marker for high grade chondrosarcomas (Fig. 4).

Interestingly, the chondrosarcoma from the patient suffering from Maffucci syndrome showed an hTERT promoter mutation, suggesting a link between these mutations and tumor progression. With regard to the five patients whose chondrosarcomas developed from a preexisting osteochondroma, one had a $h T E R T$ promoter mutation.
Table 1 Summary of patient and chondrosarcoma characteristics

\begin{tabular}{|c|c|c|c|c|}
\hline & All cases & hTERT pr & hoter & $P$-value \\
\hline & & Wild-type & Mutated & \\
\hline Total number of patients & 87 & 64 & 23 & \\
\hline Chondrosarcoma grade & & & & \\
\hline Grade 1 & 36 & $33(52 \%)$ & $3(13 \%)$ & 0.002 \\
\hline Grade 2 & 25 & $17(27 \%)$ & $8(38 \%)$ & \\
\hline Grade 3 & 26 & $14(22 \%)$ & $12(52 \%)$ & \\
\hline Tumor size $(\mathrm{cm})$ & & & & \\
\hline Average $\pm \mathrm{SD}(\min -\max )$ & $\begin{array}{l}8.2 \pm 6.1 \\
(1-36)\end{array}$ & $\begin{array}{l}8.0 \pm 5.6 \\
(1-36)\end{array}$ & $\begin{array}{l}8.9 \pm 7.4 \\
(4-17)\end{array}$ & 0.622 \\
\hline Patient age (years) & & & & \\
\hline Average $\pm \mathrm{SD}(\min -\max )$ & $\begin{array}{l}54 \pm 18.1 \\
(17-88)\end{array}$ & $\begin{array}{l}51 \pm 19.0 \\
(17-84)\end{array}$ & $\begin{array}{l}63 \pm 13.6 \\
(40-88)\end{array}$ & 0.003 \\
\hline Sex & & & & \\
\hline Male & 50 & $37(58 \%)$ & $13(57 \%)$ & 1.000 \\
\hline Female & 37 & $27(42 \%)$ & $10(43 \%)$ & \\
\hline Skeletal location & & & & \\
\hline Axial & 25 & $18(28 \%)$ & $7(30 \%)$ & 1.000 \\
\hline Appendicular & 62 & $45(72 \%)$ & $16(70 \%)$ & \\
\hline Metastasis & & & & \\
\hline Yes & 12 & $5(8 \%)$ & $7(30 \%)$ & 0.013 \\
\hline No & 75 & $59(92 \%)$ & $16(70 \%)$ & \\
\hline Local recurrence & & & & \\
\hline Yes & 21 & $15(23 \%)$ & $6(26 \%)$ & 0.783 \\
\hline No & 66 & $49(77 \%)$ & $17(74 \%)$ & \\
\hline Death at follow-up & & & & \\
\hline Dead & 13 & $5(8 \%)$ & $7(30 \%)$ & 0.013 \\
\hline Alive & 75 & $59(92 \%)$ & $16(70 \%)$ & \\
\hline Dead of disease & & & & \\
\hline Chondrosarcoma & 9 & $3(5 \%)$ & $6(26 \%)$ & 0.009 \\
\hline Alive or other death & 76 & $61(95 \%)$ & $17(74 \%)$ & \\
\hline Follow-up time (years) & & & & \\
\hline Average $\pm \mathrm{SD}(\min -\max )$ & $\begin{array}{l}4.4 \pm 4.2 \\
(0.12-31)\end{array}$ & $\begin{array}{l}4.3 \pm 4.6 \\
(0.32-31)\end{array}$ & $\begin{array}{l}4.6 \pm 2.6 \\
(0.12-11)\end{array}$ & 0.711 \\
\hline Hereditary background & & & & \\
\hline Mb Ollier & 1 & 1 & 0 & \\
\hline Mb Maffucci & 1 & 0 & 1 & \\
\hline Multiple exostosis & 1 & 1 & 0 & \\
\hline Preexisting lesion & & & & \\
\hline Exostosis & 6 & 5 & 1 & \\
\hline
\end{tabular}

$S D$ standard deviation

Significant values in bold

\section{An association between histological grade and hTERT promoter mutations in individual tumors}

To identify potential tumor subclones with $h T E R T$ promoter mutations, 14 mutated cases were stained for TERT using immunohistochemistry. We detected diffuse TERT staining in eight cases and focal TERT staining in six cases. Two cases with $h T E R T$ promoter mutations and a diffuse TERT immunoreactivity were punched in a random tumor area, and sequencing showed the mutated promoter. In two cases with focal TERT immunoreactivity we used punch biopsies from different parts of the tumor to investigate the potentiality of a relationship between immunohistochemical 
Fig. 3 Kaplan-Meier estimates of Overall survival (top), Chondrosaromca-specific survival (middle), and Metastasis-free survival (bottom) in 87 patients with conventional chondrosarcomas. Stratification includes presence of an $h T E R T$ promoter mutation (left) and chondrosarcoma histopathological grade (right)
hTERT promoter
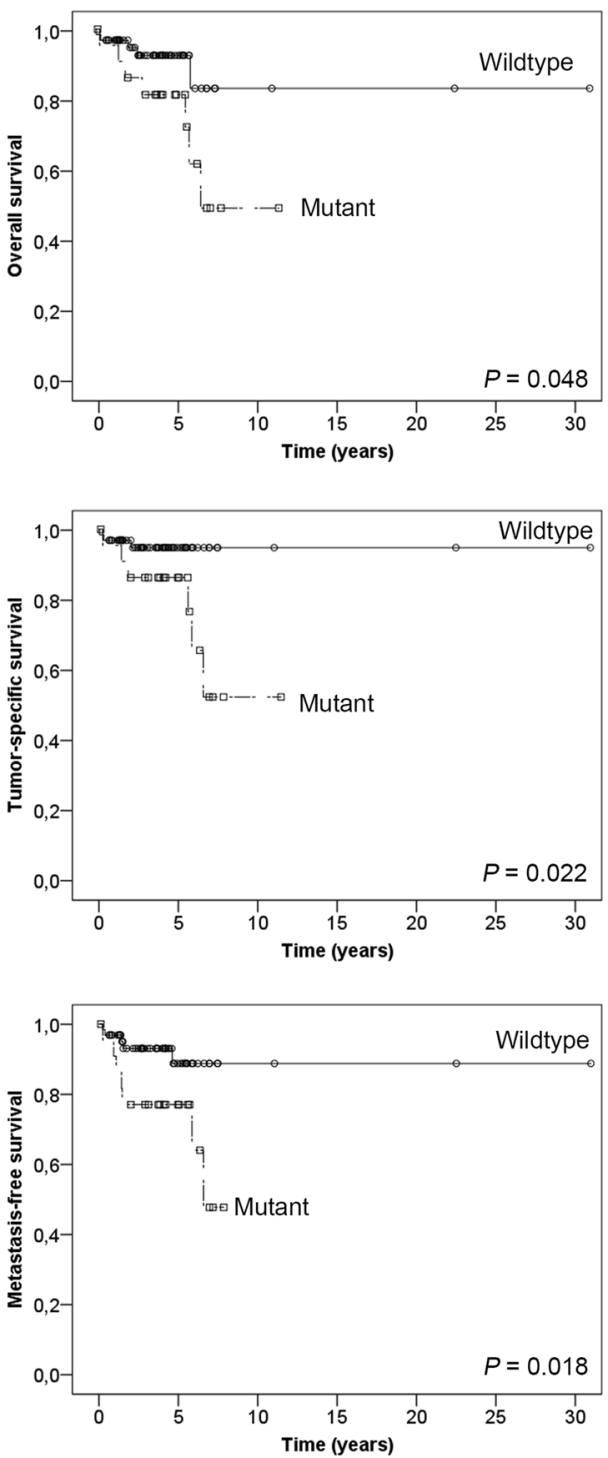
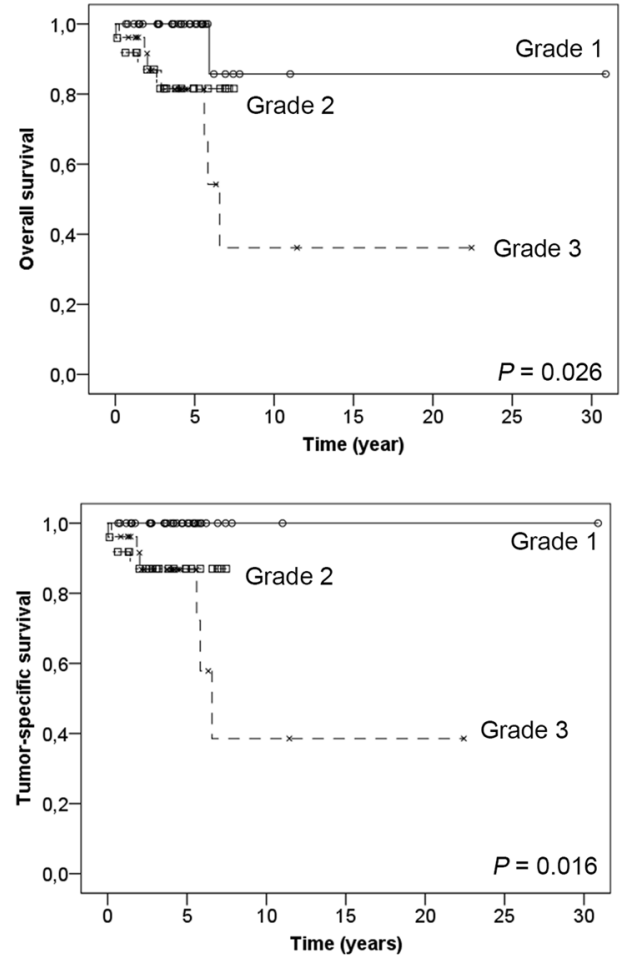

Tumor grade

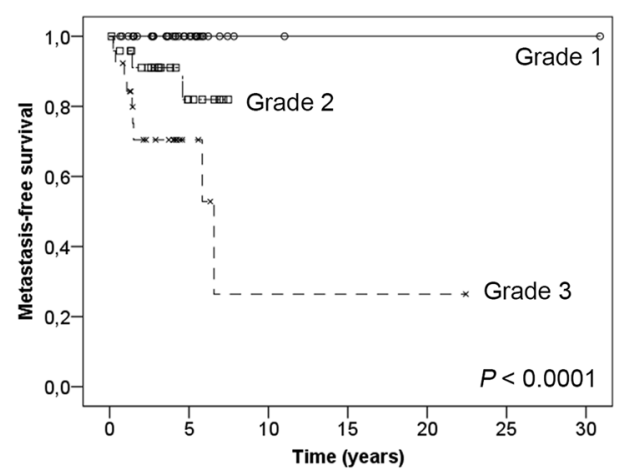

pattern, histology and $h T E R T$ promoter mutations. In these two cases, areas which expressed TERT and had a more atypical morphology harbored the hTERT promoter mutation, while low grade areas were wild type (exemplified in Fig. 5). In one of these cases, punch biopsies from a reactive spindle cell population found adjacent to the tumor showed wild-type sequence.

We also investigated TERT expression in five cases with a wild-type hTERT promoter. Diffuse TERT immunoreactivity was seen in two grade 1 tumors, focal immunoreactivity was seen in one grade 3 and negative staining was seen in a grade 2 tumor. This indicates that TERT protein expression is common in chondrosarcomas and not strongly related to tumor grade.

To further investigate the heterogeneity $h T E R T$ promoter mutations in intermediate tumors (grade 2) we investigated separate tumor blocks by analyzing punch biopsies from areas with more aggressive histology. A total of 34 punch biopsies from tumor blocks from 19 grade 2 tumors were investigated (1-3 punch biopsies per tumor based on morphology heterogeneity and material availability). Three punch biopsies from two tumors were found to harbor $h T E R T$ promoter mutations perhaps indicating that a more extensive analysis is needed to identify tumor subclones in heterogenous tumors. The chromatograms showed equivalent peaks of the wild-type and mutated alleles, further indicating that mutated subclones exists as histologically distinct population rather than a mix of mutated and wild-type tumor cells. The more extensive analysis of these 19 grade 2 chondrosarcomas could represent a potential bias. Therefore, all statistical tests were performed both with and without the two mutated cases. The small changes in $P$-values did not alter the significance of any test. 

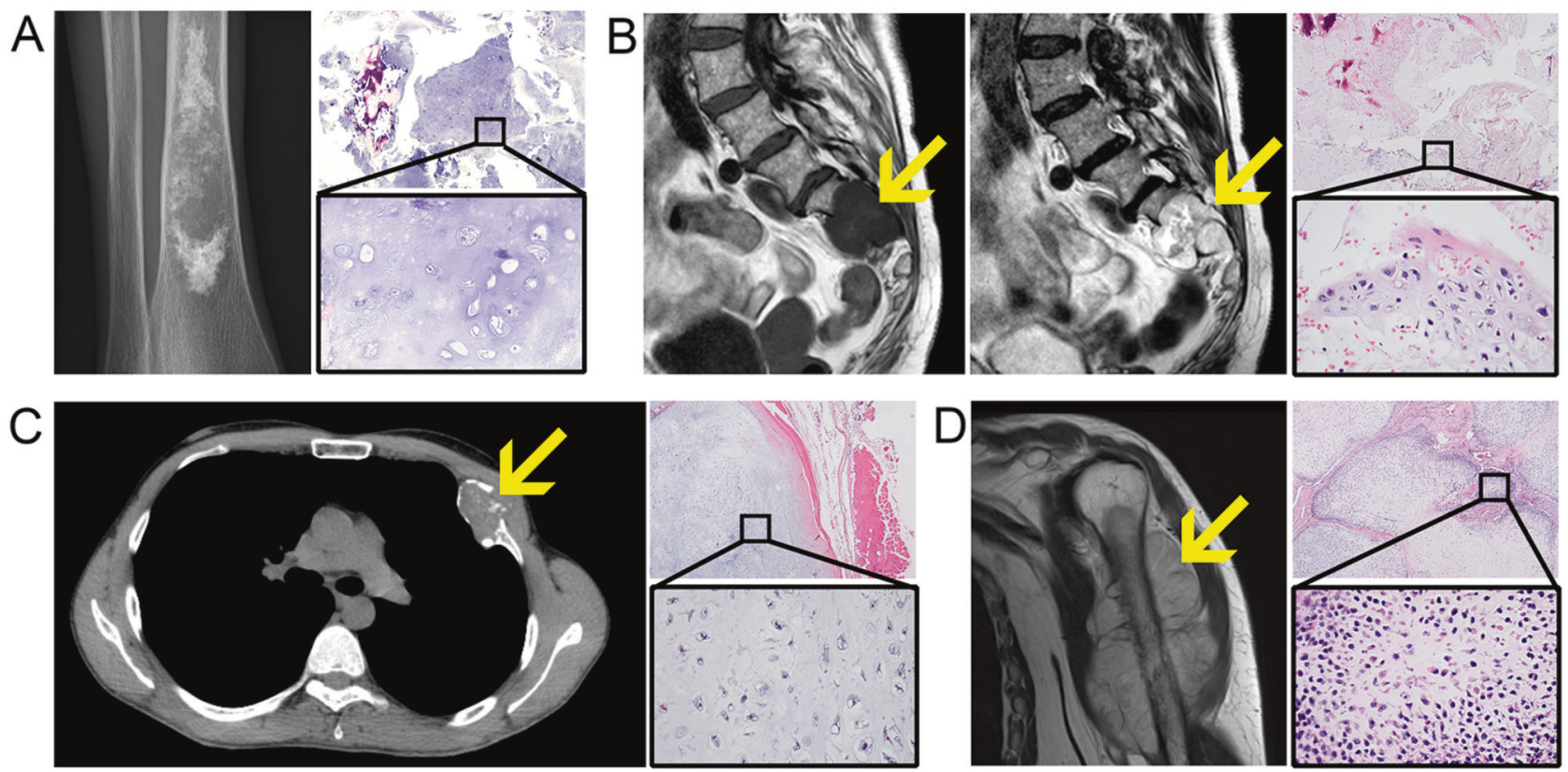

Fig. 4 Comparison between histopathology, TERT immunohistochemistry and hTERT promoter sequencing in a single chondrosarcoma with mixed histology. DNA was isolated from different areas using sterile punch biopsies (1-2). Biopsy 1 was taken from an area with grade 2 morphology (a) and TERT immunoreactivty (b). Biopsy 2 was taken from an area with grade 1 morphology (c) with no TERT immunoreactivity (d). The $-124 \mathrm{C}>\mathrm{T}$ hTERT promoter mutation was only identified in biopsy 1, while biopsy 2 showed a wild-type sequence
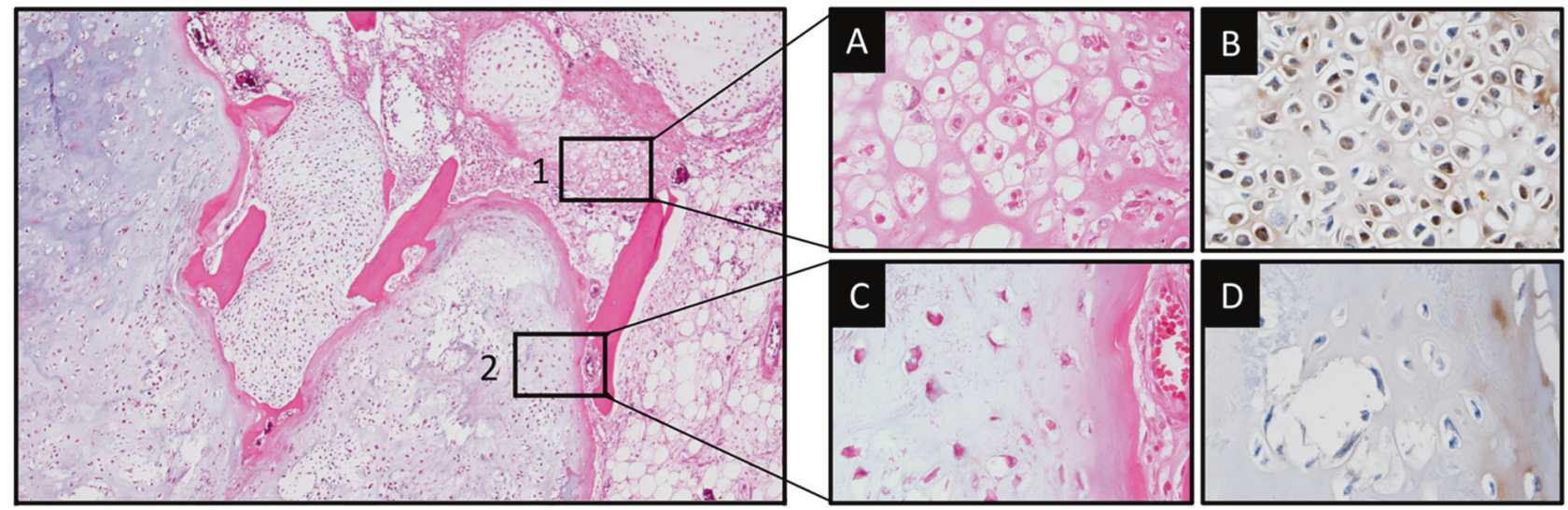

Fig. 5 Examples of imaging, histology, hTERT promoter status, and follow-up in four patients with chondrosarcoma. The arrows indicate the chondrosarcoma lesions. Chondrosarcoma, grade 1 (a). Left: Frontal X-ray of the distal tibia demonstrating a $10 \mathrm{~cm}$ lucent lesion containing typical chondroid matrix calcification (popcorn calcification). There is endosteal scalloping of the cortices and no signs of periosteal reaction. The image is consistent with a low-grade cartilaginous tumor or an enchondroma. Right: Microphotograph showing a lobulated chondroid tumor with low cellularity, limited cytological atypia and bone permeation. The tumor had no detectable hTERT promoter mutation and did not develop any recurrence or metastasis. Chondrosarcoma, grade 2 (b). Left: T2-weighted sagittal MRI of the lumbosacral column demonstrating a lobulated lesion of the S1/S2 vertebra with a high signal intensity. Several areas of low signal intensity are seen; these correspond to focal areas of dense calcification typical of cartilaginous material. Middle: T1-weighted sagittal MRI of the pelvis demonstrating a low signal intensity of the S1/S2 lesion which is typical of a cartilaginous tumor. Right: Microphotograph showing a chondroid tumor with varying cellularity, focal necrosis, pronounced cytological atypia and bone permeation. The tumor harbored a C228T hTERT promoter mutation. The patient later developed local recurrence, lung metastasis and died of the disease. Chondrosarcoma, grade 2 (c). Left: Axial CT of the thorax demonstrating an expansive lesion of the fourth left rib measuring $5 \times 4 \times 3 \mathrm{~cm}$ with an associated soft-tissue mass containing foci of calcification. The image is consistent with at least an intermediate grade central chondrosarcoma. Right: Microphotograph showing a chondroid tumor with loss of lobulated architecture, increased cellularity, and varying cytological atypia. The tumor had no detectable hTERT promoter mutation. The patient later developed local recurrence, but no distant metastasis. Chondrosarcoma, grade 3 (d). Left: T2-weighted coronar MRI of the left humerus demonstrating a high signal tumor extending from the proximal humeral metaphysis $20 \mathrm{~cm}$ distally within the diaphysis. There is a massive soft tissue tumor component growing circumferentially around the humerus which measures $10 \mathrm{~cm}$ in diameter. The MRI image is indicative of a highly aggressive tumor. Right: Microphotograph showing a highly cellular chondroid tumor with severe nuclear atypia consistent with a high-grade chondrosarcoma. The tumor harbored a C228T hTERT promoter mutation. The patient had lung metastasis at the time of diagnosis and died of the disease 


\section{Discussion}

In this study we sought to investigate if conventional chondrosarcomas harbored $h T E R T$ promoter mutations. We identified a previously characterized $-124 \mathrm{C}>\mathrm{T}$ mutation in $26 \%$ of chondrosarcomas from 87 patients, but no other promoter variants were identified. The rate of $h T E R T$ promoter mutations was increasing with tumor grade, peaking at $46 \%$ in grade 3 tumors. Furthermore, hTERT promoter mutations were significantly associated with both metastasis and death at follow-up and survival analysis showed both worse metastasis-free survival and tumor-related survival for patients with mutated tumors.

Previous studies on hTERT mutations in chondrosarcomas are limited. Two studies reported ten wild-type cases and 1/2 mutated cases, respectively [22, 23]. Without knowing the tumor grade, it is difficult to relate these data to the current study. We should point out that our extraction and sequencing protocol resulted in very good quality reads with little to no background, allowing us to detect the mutations whose peak amplitude sometimes were quite small. It is possible that other methods, e.g., mutation specific pyrosequencing or digital droplet PCR, could provide good alternatives to Sanger sequencing in the clinical routine diagnostics.

Since chondrosarcoma grading have low interobserver agreement (Kappa $0.443-0.78$ in previous publications) [3, 4], identification of easily tested DNA mutations as prognostic factors is coveted. While hTERT promoter mutations were significantly associated with the oncological outcome in this study, additional and larger studies would be required to evaluate the potential benefit of routine $h T E R T$ promoter analysis in routine chondrosarcoma diagnostics.

In previous studies, chondrosarcomas have been shown to quite frequently express the hTERT gene and harbor telomerase activity [18-21]. It is possible that activating hTERT promoter mutations are responsible for hTERT expression in at least a subset of cases. Immunohistochemistry showed TERT expression in a majority of mutated and wild-type chondrosarcomas indicating a common feature of conventional chondrosarcomas.

The true nature of $h T E R T$ mutations in chondrosarcomas remains to be fully determined. We demonstrated that the hTERT promoter mutation was solely present in the highergrade component of four chondrosarcomas with mixed histological grade. The mutation was also detected in one Maffucci-associated chondrosarcoma and one exostosisassociated chondrosarcoma, suggesting that the hTERT promoter mutation is a later genetic event that $I D H 1 / 2$ and EXT1/2 mutations. The presence of hTERT promoter mutations in 3/36 grade 1 chondrosarcomas could indicate that the mutation is present before transition to a highergrade morphology, and that the mutation is a true oncogenic driver. While $h T E R T$ promoter mutations could indicate a malignant potential, none of the mutated grade 1 chondrosarcomas (No 3) in our material reoccurred (follow up for these cases ranged 5-7 years).

Given the lack of oncological treatment and poor prognosis for metastatic chondrosarcomas, clinical trials for telomerase targeting therapies should consider to include chondrosarcoma patients.

In conclusion, we identified recurrent activating $h T E R T$ promoter mutations in chondrosarcomas which were significantly associated with tumor grade, increased risk of metastasis and worse patient survival. These results motive larger follow-up studies to validate the potential prognostic value of $h T E R T$ promoter analysis in clinical routine diagnostics.

Acknowledgements We would like to thank the Clinical Molecular Pathology laboratory at the Karolinska University Hospital in Solna for lending expertise and laboratory equipment.

Funding: This study was supported by the Cancer Society in Stockholm, the Swedish Cancer Society and the Swedish Children Cancer Foundation, the Stockholm County Council and Karolinska Institutet.

\section{Compliance with ethical standards}

Statement of ethics All patients had given written consent prior to study inclusion. The study was approved by the local ethical committee (Regionala Etikprövningsnämnden, Stockholm; registration number 2013 1979-31). The study was performed in accordance with the Declaration of Helsinki.

Conflict of interest The authors declare that they have no conflict of interest.

\section{References}

1. Fletcher CDM, Bridge JA, Lee JC. Chondrosarcomas. In: Fletcher CDM, Bridge JA, Hogendoorn PCW, et al.. (eds). WHO Classification of Tumors of Soft Tissue and Bone, 4th edn. Lyon, France: International Agency of Research on Cancer (IARC); 2013. p. 80-2.

2. Murphey MD, Flemming DJ, Boyea SR, Bojescul JA, Sweet DE, Temple HT. Enchondroma versus chondrosarcoma in the appendicular skeleton: differentiating features. Radiographics. 1998; 18:1213-37.

3. Skeletal Lesions Interobserver Correlation among Expert Diagnosticians (SLICED) Study Group. Reliability of histopathologic and radiologic grading of cartilaginous neoplasms in long bones. $\mathbf{J}$ Bone Jt Surg Am. 2007;89:2113-23.

4. Eefting D, Schrage YM, Geirnaerdt MJ, Le Cessie S, Taminiau $\mathrm{AH}$, Bovée JV, et al. Assessment of interobserver variability and histologic parameters to improve reliability in classification and grading of central cartilaginous tumors. Am J Surg Pathol. 2009;33:50-7.

5. Italiano A, Mir O, Cioffi A, Palmerini E, Piperno-Neumann S, Perrin C, et al. Advanced chondrosarcomas: role of chemotherapy and survival. Ann Oncol. 2013;24:2916-22.

6. Pansuriya TC, van Eijk R, d'Adamo P, van Ruler MA, Kuijjer ML, Oosting J, et al. Somatic mosaic IDH1 and IDH2 mutations are 
associated with enchondroma and spindle cell hemangioma in Ollier disease and Maffucci syndrome. Nat Genet. 2011;43:1256-61.

7. Amary MF, Damato S, Halai D, Eskandarpour M, Berisha F, Bonar F, et al. Ollier disease and Maffucci syndrome are caused by somatic mosaic mutations of IDH1 and IDH2. Nat Genet. 2011;43:1262-5.

8. Amary MF, Bacsi K, Maggiani F, Damato S, Halai D, Berisha F, et al. IDH1 and IDH2 mutations are frequent events in central chondrosarcoma and central and periosteal chondromas but not in other mesenchymal tumours. J Pathol. 2011;224:334-43.

9. Bovée JV, Cleton-Jansen AM, Wuyts W, Caethoven G, Taminiau $\mathrm{AH}, \mathrm{Bakker} \mathrm{E}$, et al. EXT-mutation analysis and loss of heterozygosity in sporadic and hereditary osteochondromas and secondary chondrosarcomas. Am J Hum Gen. 1999;5:689-98.

10. Björnsson J, McLeod RA, Unni KK, Ilstrup DM, Pritchard DJ. Primary chondrosarcoma of long bones and limb girdles. Cancer. 1998;83:2105-19.

11. Tarpey PS, Behjati S, Cooke SL, Van Loo P, Wedge DC, Pillay $\mathrm{N}$, et al. Frequent mutation of the major cartilage collagen gene COL2A1 in chondrosarcoma. Nat Genet. 2013;45:923-6.

12. Oshiro Y, Chaturvedi V, Hayden D, Nazeer T, Johnson M, Johnston DA, et al. Altered p53 is associated with aggressive behavior of chondrosarcoma. Cancer. 1998;83: 2324-34.

13. Tallini G, Dorfman H, Brys P, Dal Cin P, De Wever I, et al. Correlation between clinicopathological features and karyotype in 100 cartilaginous and chordoid tumours. A report from the Chromosomes and Morphology (CHAMP) Collaborative Study Group. J Pathol. 2002;196:194-203.

14. Vinagre J, Almeida A, Pópulo H, Batista R, Lyra J, Pinto V, et al. Frequency of TERT promoter mutations in human cancers. Nat Commun. 2013;4:2185.
15. Haglund F, Juhlin CC, Brown T, Ghaderi M, Liu T, Stenman A, et al. TERT promoter mutations are rare in parathyroid tumors. Endocr-Relat Cancer. 2013;22:L9-L11.

16. Horn S, Figl A, Rachakonda PS, Fischer C, Sucker A, Gast A, et al. TERT promoter mutations in familial and sporadic melanoma. Science. 2013;339:959-61.

17. Bell RJ, Rube HT, Kreig A, Mancini A, Fouse SD, Nagarajan RP, et al. The transcription factor GABP selectively binds and activates the mutant TERT promoter in cancer. Science. 2015;348:1036-9.

18. Martin JA, Forest E, Block JA, Klingelhutz AJ, Whited B, Gitelis $\mathrm{S}$, et al. Malignant transformation in human chondrosarcoma cells supported by telomerase activation and tumor suppressor inactivation. Cell Growth Differ. 2002;13:397-407.

19. Martin JA, DeYoung BR, Gitelis S, Weydert JA, Klingelhutz AJ, Kurriger $G$, et al. Telomerase reverse transcriptase subunit expression is associated with chondrosarcoma malignancy. Clin Orthop Relat Res. 2004;426:117-24.

20. Parsch D, Brassat U, Brümmendorf TH, Fellenberg J. Consequences of telomerase inhibition by BIBR 1532 on proliferation and chemosensitivity of chondrosarcoma cell lines. Cancer Invest. 2008;26:590-6.

21. Scheel C, Schaefer KL, Jauch A, Keller M, Wai D, Brinkschmidt C, et al. Alternative lengthening of telomeres is associated with chromosomal instability in osteosarcomas. Oncogene. 2001;20:3835-44.

22. Killela PJ, Reitman ZJ, Jiao Y, Bettegowda C, Agrawal N, Diaz LA, et al. TERT promoter mutations occur frequently in gliomas and a subset of tumors derived from cells with low rates of selfrenewal. Proc Natl Acad Sci USA. 2013;110:6021-6.

23. Saito T, Akaike K, Kurisaki-Arakawa A, Toda-Ishii M, Mukaihara $\mathrm{K}$, Suehara $\mathrm{Y}$, et al. TERT promoter mutations are rare in bone and soft tissue sarcomas of Japanese patients. Mol Clin Oncol. 2013;4:61-4. 